TITLE:

\title{
Absorption-free optical control of spin systems: The quantum Zeno effect in optical pumping
}

$\operatorname{AUTHOR}(S):$

Nakanishi, T; Yamane, K; Kitano, M

\section{CITATION:}

Nakanishi, T ...[et al]. Absorption-free optical control of spin systems: The quantum Zeno effect in optical pumping. PHYSICAL REVIEW A 2002, 65(1): 013404.

ISSUE DATE:

2002-01

URL:

http://hdl.handle.net/2433/39821

RIGHT:

Copyright 2002 American Physical Society 
PHYSICAL REVIEW A, VOLUME 65, 013404

\title{
Absorption-free optical control of spin systems: The quantum Zeno effect in optical pumping
}

\author{
T. Nakanishi, K. Yamane, and M. Kitano \\ Department of Electronic Science and Engineering, Kyoto University, Kyoto 606-8501, Japan
}

(Received 19 February 2001; published 13 December 2001)

\begin{abstract}
We show that atomic spin motion can be controlled by circularly polarized light without light absorption in the strong pumping limit. In this limit, the pumping light, which drives the empty spin state, destroys the Zeeman coherence effectively and freezes the coherent transition via the quantum Zeno effect. It is verified experimentally that the amount of light absorption decreases asymptotically to zero as the incident light intensity is increased.
\end{abstract}

DOI: 10.1103/PhysRevA.65.013404

PACS number(s): 32.80.Bx, 03.65.Xp, 42.50.-p

The quantum Zeno effect is known as the suppression of unitary time evolution caused by decoherence in quantum systems $[1,2]$. The decoherence is provided by measurement, interactions with the environment, stochastic fields, and so on. It is interesting in the sense that the coherent, unitary evolution can be canceled by decoherent processes in a probability conserving manner. If we look at the effect from the frame that moves so as to cancel the unitary evolution, the system, otherwise stationary, is guided by the decoherence processes. Using this inverse Zeno effect, we can control quantum systems with incoherent processes without the loss of probability [3].

In this paper, we will show that the quantum Zeno effect plays a crucial role in the optical pumping scheme. Though not widely recognized, the light-induced decoherence has a strong influence on the spin dynamics. Optical pumping is a useful method to polarize atoms [4]. Let us consider a fourlevel atom with a $J=1 / 2$ ground state and a $J^{\prime}=1 / 2$ excited state. In order to polarize the atomic spin in the ground state, we apply a $\sigma_{+}$pumping light beam that is propagated in the $z$ direction and tuned to the transition between the ground state and the excited state. The only spin-down atoms $\left(m_{J}\right.$ $=-1 / 2$ ) are excited by absorbing the pumping light, and relax either to the spin-up state $\left(m_{J}=1 / 2\right)$ or to the spindown state in the ground level. On the other hand, the spin-up atoms cannot absorb the pumping light. When the pumping light is strong enough, all the spin-down atoms are pumped out to the spin-up state. Once the spins are completely polarized, the atomic ensemble does not absorb the pumping light anymore.

However, in the presence of a magnetic field transverse to the pumping light, the polarized spins are flipped back to the spin-down state. The magnetic field tends to equalize the population of the spin-down state with that of the spin-up state through the precession. Therefore, the absorption of the pumping light is needed to compensate the transition caused by the magnetic field.

But it has been found that the spin polarization can be retained against the transverse magnetic field without any absorption of the pumping light [5]. At first sight, it may seem contradictory, but the phenomenon can be understood by considering the decoherence caused by the pumping light. The spin precession itself is suppressed by the light-induced decoherence via the quantum Zeno effect, which accompanies no light absorption.
In terms of the normal interpretation of the quantum Zeno effect that the time evolution is suppressed by the frequent measurements, the transmission monitoring of the polarized light plays the role of measurements, from which we can infer the spin direction. The absence of absorption is clear evidence that all the spin are in the up state. By performing the null measurements frequently or continuously, one can freeze the spin dynamics. This is a kind of interaction-free measurement [6].

When we apply a $\sigma_{+}$pumping light beam in the $z$ direction under an external magnetic field $\boldsymbol{B}_{0}$, the normalized magnetic moment $\boldsymbol{m}$ obeys the Bloch equation,

$$
\frac{d \boldsymbol{m}}{d t}=\boldsymbol{m} \times \boldsymbol{\Omega}_{0}-\Gamma \boldsymbol{m}-P\left(\boldsymbol{m}-\boldsymbol{e}_{z}\right),
$$

where $\left(\boldsymbol{e}_{x}, \boldsymbol{e}_{y}, \boldsymbol{e}_{z}\right)$ are the Cartesian unit vectors. The first term on the right-hand side represents the spin precession around $\boldsymbol{B}_{0}$ at the angular frequency $\left|\boldsymbol{\Omega}_{0}\right|=\gamma_{g}\left|\boldsymbol{B}_{0}\right|$ with the gyromagnetic ratio $\gamma_{g}$. The second term represents the spin relaxation by which the magnitude of the magnetic moment is decreased exponentially at the rate $\Gamma$. The relaxation is not an essential element for the quantum Zeno effect but we cannot ignore it in the actual experiment. The third term represents the optical pumping toward $\boldsymbol{m}=\boldsymbol{e}_{z}$ with the pumping rate $P$, which is proportional to the light intensity $I$.

The steady-state solution to Eq. (1) is

$$
\boldsymbol{m}=-\frac{P \Omega_{0}}{(P+\Gamma)^{2}+\Omega_{0}^{2}} \boldsymbol{e}_{x}+\frac{P(P+\Gamma)}{(P+\Gamma)^{2}+\Omega_{0}^{2}} \boldsymbol{e}_{z},
$$

where we have assumed that the magnetic field is applied in the $y$ direction; $\boldsymbol{\Omega}_{0}=\boldsymbol{\Omega}_{0} \boldsymbol{e}_{y}$.

For the strong pumping limit, i.e., for $P \gg \Omega_{0}, P \gg \Gamma$, we have $\boldsymbol{m} \sim \boldsymbol{e}_{z}-O\left(\Omega_{0} / P\right) \boldsymbol{e}_{x}$. This means that in this limit all the spin are polarized in the $z$ direction even in the presence of the transverse magnetic field.

The key point of the optical pumping is the selective population transfer via excited states and a population distribution far from equilibrium in the ground states can be achieved. Therefore, one might think that in the above situation the spin polarization is maintained by the repumping (population transfer) of the atoms flipped by the transverse magnetic field. However, as will be shown below, this is not the case. The spin flip itself is suppressed by the quantum 
Zeno effect induced by the pumping light. By exciting the empty state (the spin-down state in this case), the pumping light destroys the Zeeman coherence and results in the Zeno effect.

One can distinguish the two scenarios by observing the absorption of the pumping light. In the former case, we should see some absorption corresponding to the repumping of the spin flipped by the magnetic field. In the latter case, however, we will see no absorption, at least in principle, because the spin rotation is suppressed owing to the Zeno effect. The Zeno effect can be realized without absorption when the decoherence is provided by the excitation of the empty state.

We will separate the pumping term in the Bloch equation (1) into the pumping (population transfer) effect and the decoherence effect. The pumping effect appears in the $z$ component as

$$
\left.\frac{d m_{z}}{d t}\right|_{\text {pump }}=P\left(1-m_{z}\right),
$$

which represents the population transfer from the spin-down state to the spin-up state. The population transfer necessarily accompanies optical absorption (and subsequent reemission). For the completely polarized state, $m_{z} \sim 1$, this term has little effect on the spin dynamics.

On the other hand, the decoherence effect appears in the $x, y$ components as

$$
\left.\frac{d m_{x, y}}{d t}\right|_{\text {pump }}=-P m_{x, y} .
$$

Note that both $m_{x}$ and $m_{y}$ correspond to the coherence $\rho_{+-}$ between the spin-up $|+\rangle$ and spin-down $|-\rangle$ states. The coherence decays at the rate $\Gamma+P$ in the presence of the pumping light. Therefore, Eq. (4) represents the decoherence. This decoherence causes the suppression of the spin precession via the quantum Zeno effect. It does not accompany optical absorption and works even when $m_{z} \sim 1$.

The pumping rate $P$ is connected with the pumping light intensity $I$ as

$$
P=\eta \sigma \frac{I}{\hbar \omega}
$$

where $\eta, \sigma$, and $\hbar \omega$ are the pumping efficiency, the absorption cross section, and the photon energy, respectively. The absorption coefficient $\alpha$ is defined as

$$
\alpha=N \sigma \frac{1}{2}\left(1-m_{z}\right),
$$

where $N$ is the atomic density. Substituting $m_{z}$ in Eq. (6) by Eq. (2), we obtain

$$
\alpha=\frac{N \sigma}{2} \frac{\Gamma(\Gamma+P)+\Omega_{0}^{2}}{(\Gamma+P)^{2}+\Omega_{0}^{2}} .
$$

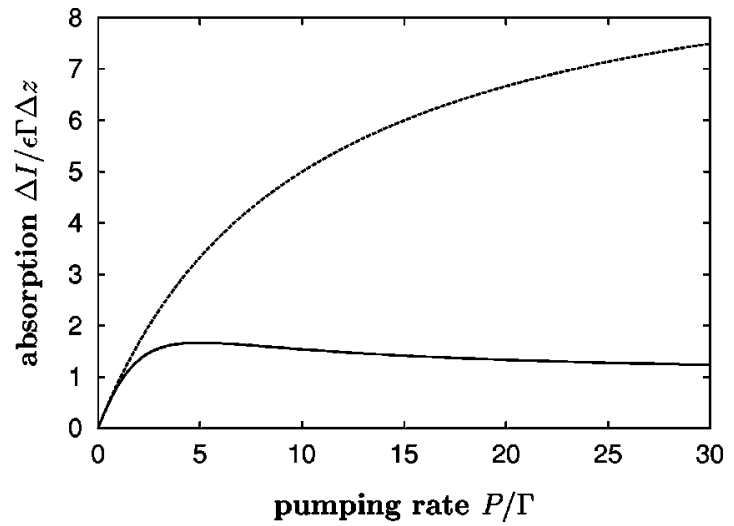

FIG. 1. The solid line represents the theoretical absorption. The dashed line is the absorption curve obtained by neglecting the terms related to the quantum Zeno effect. The optical pumping rate $P$ and the angular frequency of the precession $\Omega_{0}$ are normalized by the relaxation rate $\Gamma$, and these lines are calculated for $\Omega_{0} / \Gamma=3$. The absorption rate is also normalized to satisfy $\lim _{P / \Omega_{0} \rightarrow \infty} \Delta I / I_{0}=1$.

For optically thin cases, i.e., $\alpha \Delta z \ll 1$, the absorption of the pumping light $\Delta I$ is given by $\Delta I=I \alpha \Delta z$, where $\Delta z$ is the length of the cell. With Eqs. (5) and (7), we obtain

$$
\Delta I=\epsilon P \frac{\Gamma(\Gamma+P)+\Omega_{0}^{2}}{(\Gamma+P)^{2}+\Omega_{0}^{2}} \Delta z
$$

where we introduced $\epsilon=N \hbar \omega / 2 \eta$ for simplicity.

Here we see that $\Delta I$ monotonically increases with $P$ when $\Gamma>\Omega_{0}$, i.e., when the relaxation dominates the spin precession. However, for $\Gamma<\Omega_{0}, \Delta I$ increases up to a point $P$ $=\left(\Omega_{0}^{2}+\Gamma^{2}\right) /\left(\Omega_{0}-\Gamma\right)$, then $\Delta I$ starts to decrease. In Fig. 1 we plotted $\Delta I$ as a function of $P$ in the case of $\Gamma<\Omega_{0}$. The absorption approaches asymptotically a specific value:

$$
\lim _{P / \Omega_{0} \rightarrow \infty} \Delta I=\epsilon \Gamma \Delta z
$$

Thus, in the ideal condition where the intrinsic relaxation can be neglected, one can suppress the spin evolution by the pumping light with no absorption. Notice that Eq. (9) is independent of the precession frequency $\Omega_{0}$ and is proportional to the relaxation rate $\Gamma$. The relaxation is an incoherent process and cannot be suppressed by the Zeno effect. On the other hand, the precession is a coherent process and can be suppressed.

The dashed line in Fig. 1 represents the hypothetical curve for which the decoherence presented by Eq. (4) is omitted. The actual absorption (the solid line) is much smaller than the dashed line. Moreover, the solid line is qualitatively different from the dashed line in that it has a maximum point and decreases with $P$ thereafter. If the spin precession were not suppressed by the pumping light, we would have a normal saturated absorption profile like the dashed line in Fig. 1. This is because the polarized spins continue to precess at a certain rate and absorb the pumping light. The fact that the absorption decreases with $P$ is a clear evidence for the suppression of the precession by the pumping-induced decoher- 


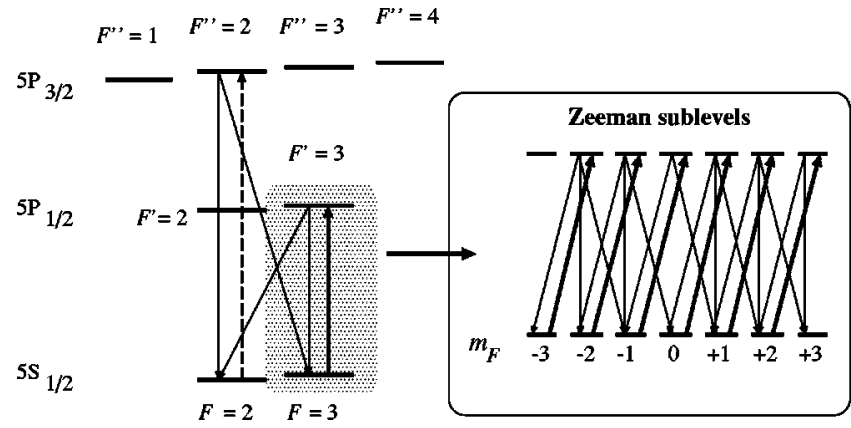

FIG. 2. ${ }^{85} \mathrm{Rb}$ energy diagram. The arrows represent optical pumping (thick lines), repumping (dashed line), and the spontaneous emission (solid lines).

ence. The reduction of the absorption is a peculiar feature originating from the quantum Zeno effect.

We use ${ }^{85} \mathrm{Rb}$ atoms to verify the absorption-free feature experimentally. The energy diagram for ${ }^{85} \mathrm{Rb}$ is illustrated in Fig. 2. A laser for the optical pumping is tuned to the transition frequency between $F=3$ and $F^{\prime}=3$ in the $D_{1}$ line $\left(5 S_{1 / 2} \rightarrow 5 P_{1 / 2}\right)$. The circularly polarized pumping light $\left(\sigma_{+}\right)$induces the transitions satisfying $\Delta m_{F}=+1$, and eventually populates all the atoms to the Zeeman sublevel $m_{F}$ $=3$ in the absence of the transverse magnetic field.

The pumping cycle is not closed within the transition between $F=3$ and $F^{\prime}=3$; some of excited atoms in $F^{\prime}=3$ drop to the $F=2$ level and are accumulated in that level. In order to prevent the hyperfine pumping we prepared another laser which was tuned to the transition frequency from the $F=2$ level in the ground state to the $5 P_{3 / 2}$ state $\left(D_{2}\right.$ line). When the laser intensity is strong enough to repump the atoms in the $F=2$ level, the pumping cycle is effectively closed (meshed region). The intensity of the repumping light must be comparable to, or more than, that of the pumping laser.

In our experimental condition, the absorption coefficient for the pumping light is so small that we had to measure it with some precision. We used a differential absorption method. When the repumping light is turned on, $\mathrm{Rb}$ atoms show the expected absorption of the pumping light. On the other hand, when the repumping light is turned off, due to the hyperfine pumping, there remains few atoms in the $F$ $=3$ level, and the absorption of the pumping light becomes negligibly small. The difference in the transmitted intensity of the pumping light between the two cases corresponds to the absorption $\Delta I$ to be measured.

Our experimental setup is illustrated in Fig. 3. We used an external-cavity diode laser with maximum output power 40 $\mathrm{mW}$ for pumping $\left(D_{1}\right.$ line). A similar system was prepared for repumping $\left(D_{2}\right.$ line). The power of the pumping laser can be adjusted using a half-wave plate and a polarizing beam splitter. The pumping and repumping beams were aligned so as to well overlap each other in the Rb cell. The cell $(2.2 \mathrm{~cm}$ in length and $2.0 \mathrm{~cm}$ in diameter) is kept at room temperature. The cross section of the pumping beam, $A_{p}$, is about $0.12 \mathrm{~cm}^{2}$, and that of the repumping beam, $A_{r}$, is about 0.36 $\mathrm{cm}^{2}$.

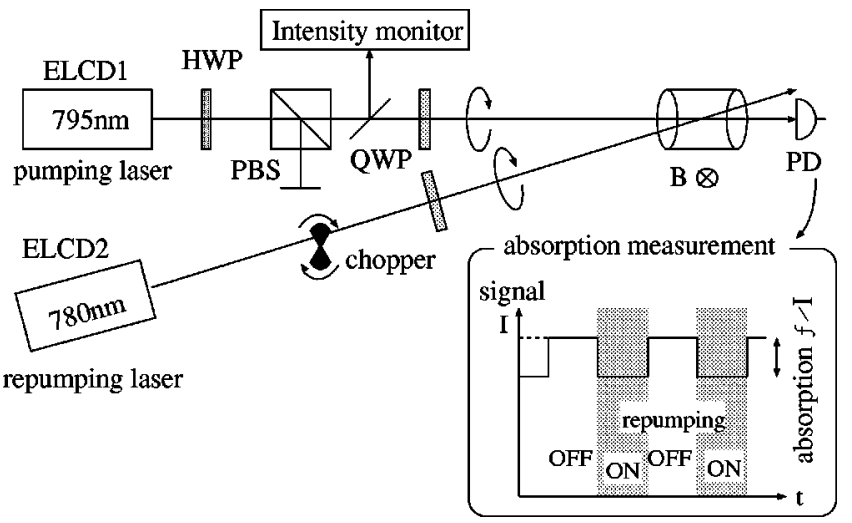

FIG. 3. Experimental setup. The inset shows the output signal of the photodetector (PD). Chopping of the repumping laser results in the rectangular wave whose amplitude corresponds to the absorption of the pumping light.

It is important to minimize the spin relaxation. The primary factor of the relaxation is the diffusion of the atoms to the cell wall. In order to suppress the atomic diffusion we used nitrogen as buffer gas at $8.0 \mathrm{kPa}$. A uniform magnetic field can be applied to the Rb cell by three pairs of Helmholtz coils.

As shown in Fig. 3, we measure the transmitted light intensity by using a photodiode (PD) while chopping the repumping laser by a mechanical chopper. We have a rectangular wave form whose amplitude corresponds to the absorption of the pumping light (see the inset). The chopping frequency $(91 \mathrm{~Hz})$ is smaller than the characteristic frequencies of the system, $P, \Omega_{0}$, and $\Gamma$ ( $>$ several kilohertz).

In order to increase the pumping rate effectively, the transmitted light through the $\mathrm{Rb}$ cell is reflected back by a mirror so that the reflected light takes almost the same path. It should be noted that this does not mean that only the light path $\Delta z$ interacting with $\mathrm{Rb}$ atoms is doubled. By overlapping the beams we can also double the pumping rate $P$ effectively. Then Eq. (5) is modified as

$$
P=\frac{2 \eta \sigma}{\hbar \omega} I
$$

and the absorption (8) is replaced by

$$
\Delta I=I \alpha(2 \Delta z)=\epsilon P \frac{\Gamma(\Gamma+P) \Omega_{0}^{2}}{(\Gamma+P)^{2}+\Omega_{0}^{2}} \Delta z .
$$

Figure 4 shows the experimentally obtained absorption curve. The power of the pumping laser incident to the $\mathrm{Rb}$ cell was varied from 0 to $28 \mathrm{~mW}$, and the power of the repumping laser was fixed at $32.8 \mathrm{~mW}$. The strength of applied magnetic field was $2 \mu \mathrm{T}\left(\Omega_{0} \sim 9 \mathrm{kHz}\right)$. Fitting the results to the theoretical curve (solid line), we estimated the relaxation rate to be about $3 \mathrm{kHz}$. Owing to the differential absorption technique, we were able to measure small variation in the absorption constant $(\Delta I / I<2 \%)$ with high accuracy $(<0.1 \%)$.

The result shows that the absorption decreases when the pumping power is over about $10 \mathrm{~mW}$. The decrease in the 


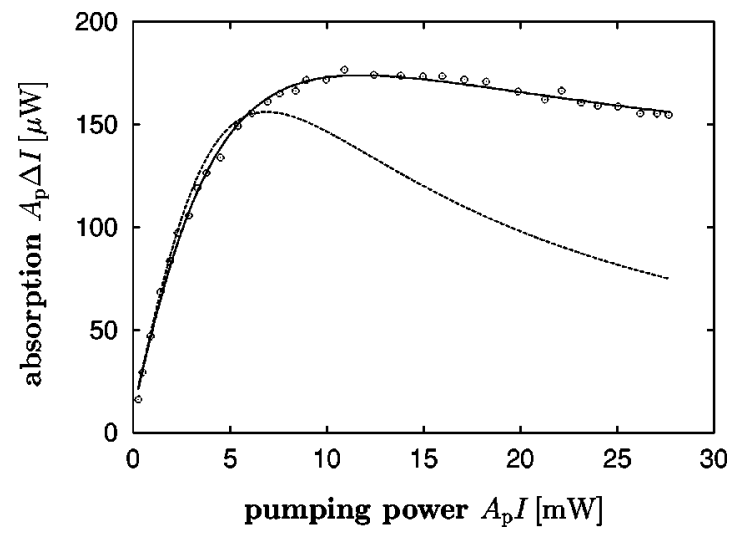

FIG. 4. Experimental results $(\odot)$, theoretical line fitted with the results (solid line), and theoretical line without relaxation (dashed line). Applied magnetic field was $2 \mu \mathrm{T}$.

absorption was more than fluctuations (a few $\mu \mathrm{W}$ ). We emphasize repeatedly that the decrease in the absorption is qualitatively different either from linear absorption or from saturated absorption. This phenomenon is induced by the Zeno suppression of the spin precession. The theoretical curve shows good agreement with the experimental result.

As mentioned before, in the strong pumping limit the spin precession can be suppressed completely by the quantum Zeno effect, but the spin relaxation still persists. The dashed line in Fig. 4 shows the absorption curve for which the absorption related to the relaxation is subtracted. This line declines more rapidly with the increase of pumping power, and is supposed to tend to zero. If we were to eliminate the intrinsic relaxation, we would have the pronounced decrease in the absorption.
We can increase buffer gas pressure to reduce the relaxation due to the diffusion. However, the spin relaxation by the collision increases instead, and we cannot expect drastic improvement. A considerable improvement will be expected by cooling the atoms. But the laser cooling is usually performed under inhomogeneous magnetic fields, so we have to turn off the magnetic fields as well as the cooling laser beams. Another possible method is the use of a glass cell containing dense ${ }^{4} \mathrm{He}$ buffer gas. At the temperature blow 2.1 $\mathrm{K}$, the inner wall is coated with liquid ${ }^{4} \mathrm{He}$ films and the spin relaxation time is significantly reduced [7]. This method has the advantage that it is free from magnetic fields and gravity. It is reported that the spin polarization time can be extended to as long as $\sim 60 \mathrm{~s}$.

The suppression of the coherent transition by the optical pumping is applicable to the quantum-state control of highly degenerate multilevel systems such as in quantum logic gates. In order to prevent the population diffusion to undesired levels, we can optically pump these empty levels selectively. Moreover, we can attain active control of spin states by using the inverse Zeno effect. By changing the pumping direction or the polarization continuously, we can change the spin direction arbitrarily. This is similar to the coherent population trapping or the adiabatic passage with the dark state [8]. A recent paper [9] presents the application to the preparation of quantum state in superposition.

Note added. A related paper by Mølhave and Drewsen [10] was previously published.

We wish to thank T. Ikushima for helpful discussions. This research was supported by the Ministry of Education, Culture, Sports, Science and Technology in Japan under a Grant-in-Aid for Scientific Research No. 11216203 and No. 11650043.
[1] B. Misra and E. C. G. Sudarshan, J. Math. Phys. 18, 756 (1977).

[2] W. M. Itano, D. J. Heinzen, J. J. Bollinger, and D. J. Wineland, Phys. Rev. A 41, 2295 (1990).

[3] T. P. Altenmüller and A. Schenzle, Phys. Rev. A 48, 70 (1993).

[4] W. Happer, Rev. Mod. Phys. 44, 169 (1972).

[5] M. Kitano, K. Yamane, and T. Ikushima, Phys. Rev. A 59, 3710 (1999).
[6] P. Kwiat, H. Weinfurter, T. Herzog, and A. Zeilinger, Phys. Rev. Lett. 74, 4763 (1995).

[7] A. Hatakeyama, K. Oe, K. Ota, S. Hara, J. Arai, and T. Yabuzaki, Phys. Rev. Lett. 84, 1407 (2000).

[8] M. Kitano, Phys. Rev. A 56, 1138 (1997).

[9] A. Luis, Phys. Rev. A 63, 052112 (2001).

[10] K. Mølhave and M. Drewsen, Phys. Lett. A 268, 45 (2000). 